\title{
Two Case Reports of Madelung's Disease: CT Finding.
}

\author{
Chanpen Buranachokpaisan, MD. \\ Department of Radiology, Buddachinnaraj Hospital, Phitsanulok, Thailand.
}

\begin{abstract}
Madelung's disease or Benign symmetric lipomatosis is the rare disease that is the one of differential diagnosis of palpable neck mass that easily diagnosis by CT imaging. We report two cases of this condition and review the radiological findings.
\end{abstract}

Key words: Maldelung's diseases, Multiple symmetric lipomatosis (MSL), Launois-Bensaude Syndrome

\section{Introduction}

Madelung's disease is a rare condition characterized by the growth of fatty masses in different parts of the body in a very specific pattern or distribution. The disease frequently affects men with a Mediterranean origin between the ages of 30 and 70 with a history of alcohol abuse. Non-alcoholics and women are also affected. The signs and symptoms are varying greatly from person to person. The disease may cause cosmetic deformity, movement limitation, and peripheral neuropathy. The serious complication is airway obstruction. The disease is benign condition that unknown cause but most strongly associated with alcohol consumption. However, there is a reported case in which became liposarcoma. The treatment might include reduced alcoholic intake, correct metabolic conditions and surgery to remove lipomas in cases of mass effect or cosmetic concerned ${ }^{(1)}$. We report two cases of the Madelung's disease in Asian countries and review the radiological findings.

\section{Case report}

\section{Case 1}

A 43-year old man presented with painless left neck mass with progressive growth for 1 year. The physical examination revealed an ill-defined mass at left upper neck and submandibular area approximately $6 \mathrm{~cm}$ and an ill-defined mass at right upper neck about $3 \mathrm{~cm}$, other ENT examinations are within normal limits. No neurological deficit or muscular weakness is noted. Past history of heavy alcohol consumption for 20 years and was a nonsmoker. No other underlying disease was noted.

Laboratory test results were the following: $\mathrm{Hb}$ $12.8 \mathrm{~g} / \mathrm{dl}$; Hct 40\%; MCV $82.3 \mathrm{fl}$; MCH $26.2 \mathrm{pg}$; MCHC 31.8 g/dl; RDW 15.5\%; WBC 8540/mm³ (Neu $67 \%$, mono8\%, lymph $23 \%$, Eos $1 \%$ ) ; platelet 222,000/mm ; Anti-HIV negative. A liver ultrasound showed mild liver enlargement with diffuse fatty infiltration.

Multi-Detector Computed Tomography (MDCT) scan showed excessive fat deposit diffusely in sub- 


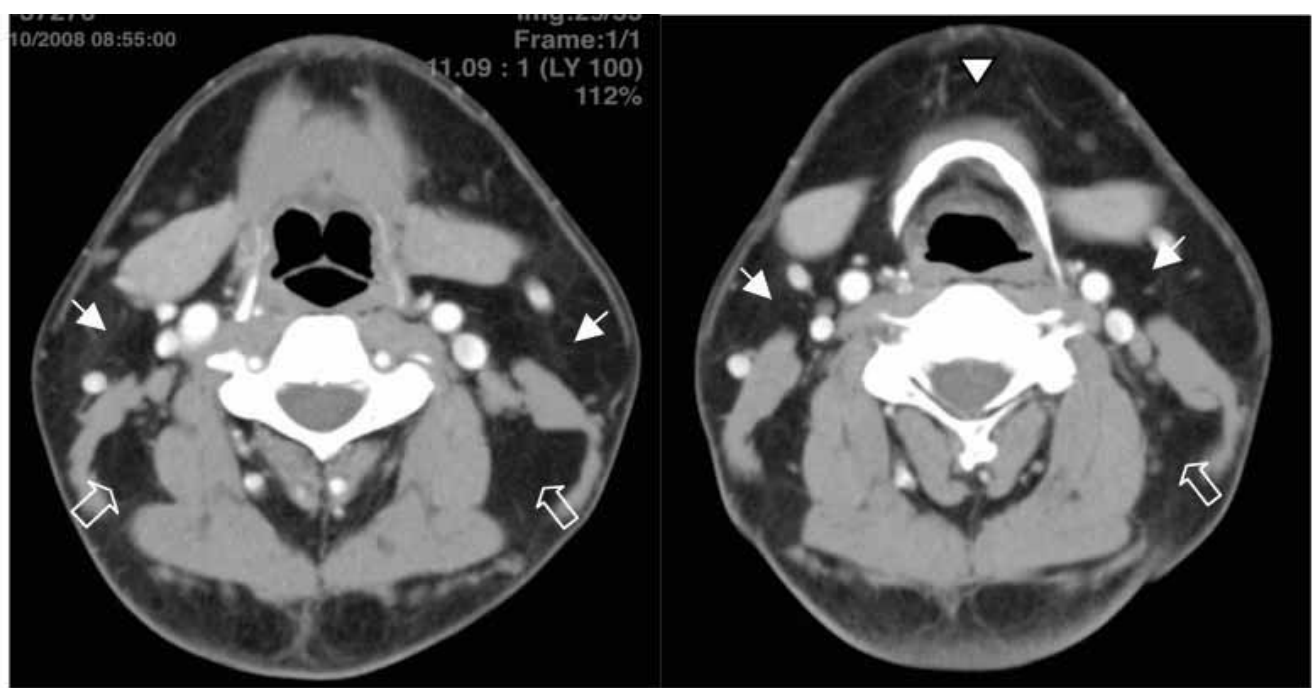

Fig. 1A

Fig. 1B

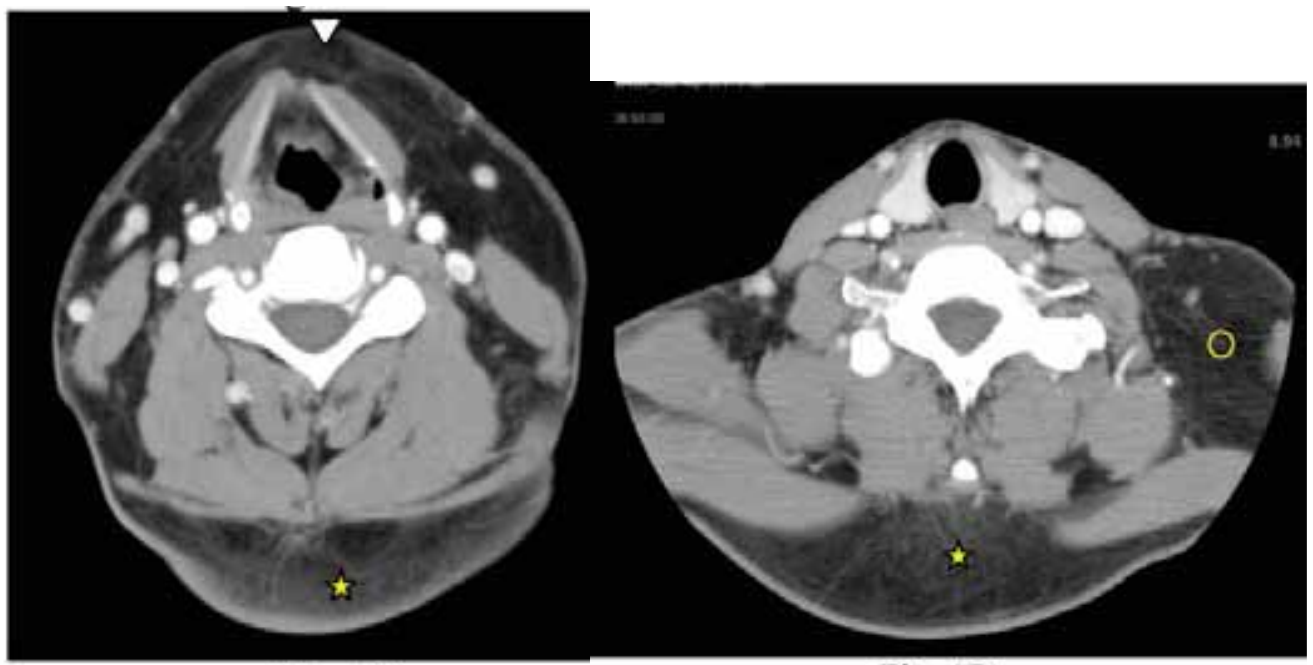

Fig. 1C

Fig. 1D

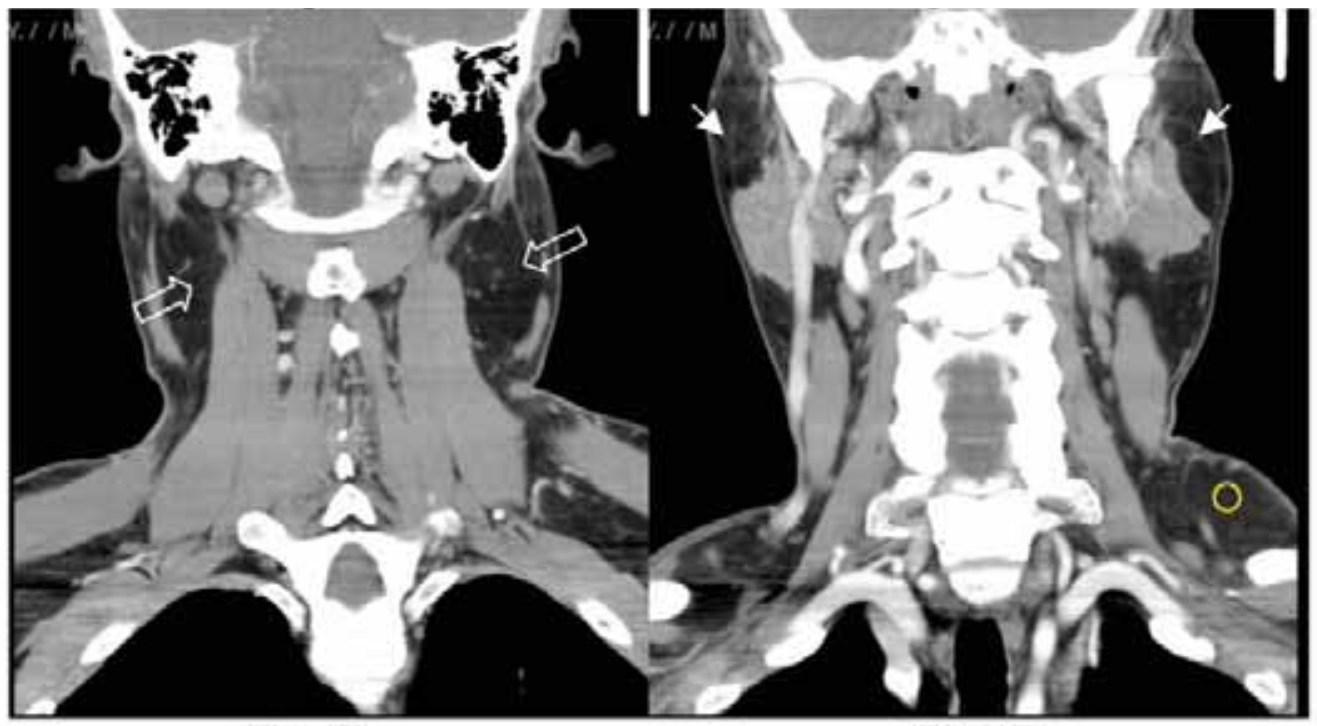

Fig. 1E

Fig. 1F 


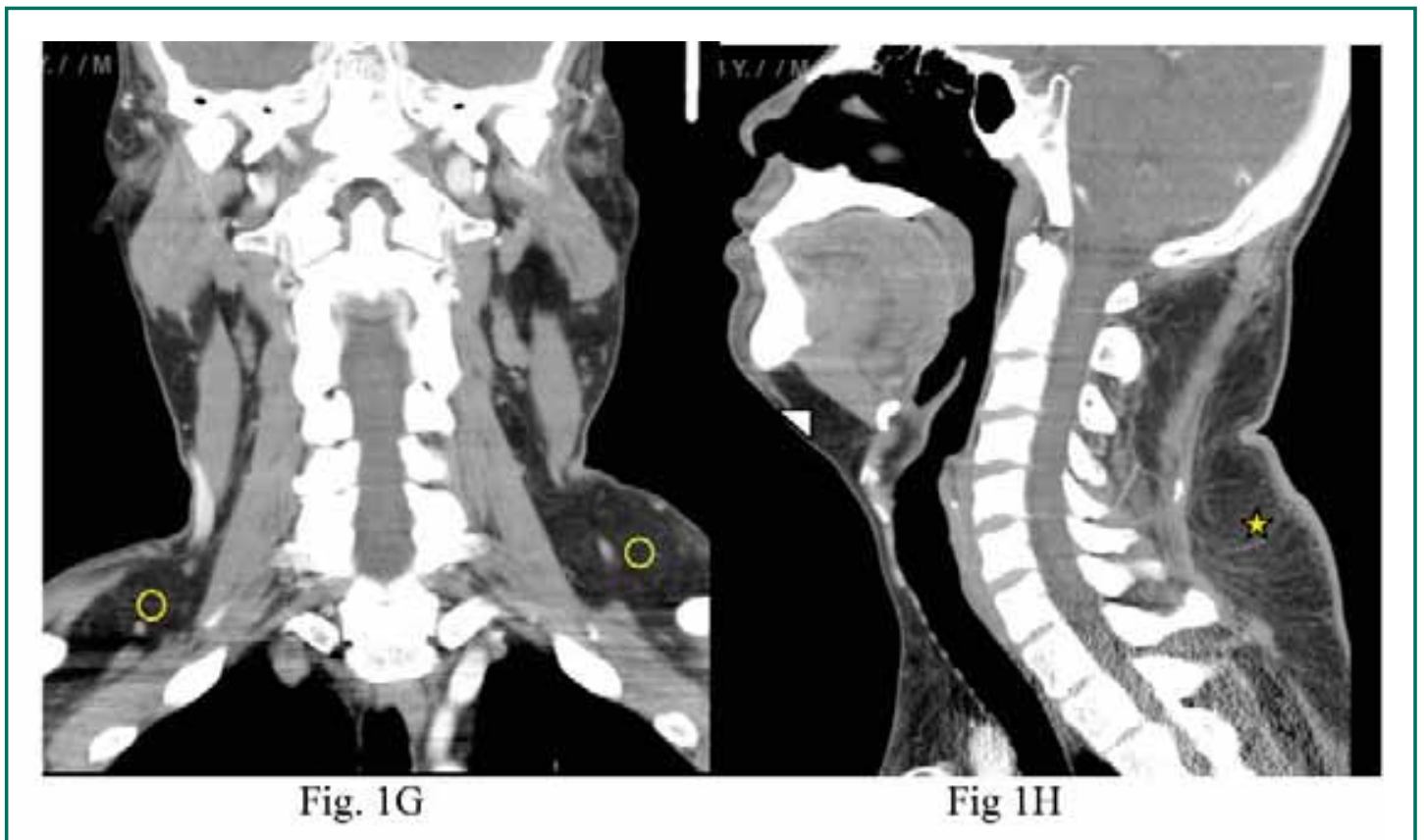

Figure.1 1A - 1D axial views and Figure. 1E - 1H coronal reconstruction of CT study showed excessive fat deposit diffusely in subcutaneous tissue of both cheeks and bilateral carotid spaces (small white arrow), the anterior part of neck (arrow head), above and below bilateral sternocleidomastoid muscles (blank arrow), paraspinal muscles, bilateral posterior triangles (yellow circle) and posterior aspect of the neck (star), predominately on the left sided.

cutaneous tissue of the anterior part of neck (suprahyoid, infrahyoid, interclavicular and superior mediastinal regions), both cheeks, bilateral carotid spaces, above and below bilateral sternocleidomastoid muscles, bilateral posterior triangles, paraspinal muscles and posterior aspect of the neck and bilateral supraclavicular regions, predominately at left sided. A clinical diagnosis of Madelung's disease type I was made. (Figure $1 \mathrm{~A}-\mathrm{H}$ )

Since our patient was asymptomatic, no surgical treatment was proposed. He was started on lipid-lowering therapy and referred to an alcohol detoxification program.

\section{Case 2}

A 47-year old man presented with bilateral cervical neck masses for 2 years. No dysphagia, odynophagia or weight loss was noted. The patient had a history of longstanding alcohol consumption more than 10 years and was a nonsmoker. The physical examination showed generalized soft tissue masses at both cervical necks. The ENT examination was normal. Both lungs were clear. No neurological deficit or myelopathy.

The Laboratory results were only; BUN = 12 $\mathrm{mg} / \mathrm{dl}, \mathrm{Cr}=0.75 \mathrm{mg} / \mathrm{dl}$ and $\mathrm{eGFR}=118.84 \mathrm{cc} / \mathrm{min}$.

Multi-Detector Computed Tomography (MDCT) scan revealed generalized excessive fat density deposition at chin, cervical paraspinal and surrounding all anterior, lateral and posterior necks without definite mass, cyst or calcification. The clinical diagnosis of Madelung's disease type I was also noted (Fig 2A-F). This patient was also asymptomatic, just reassuring and no surgical treatment was performed. He was started in a reduced lipid and alcohol intakes and exercise program. 


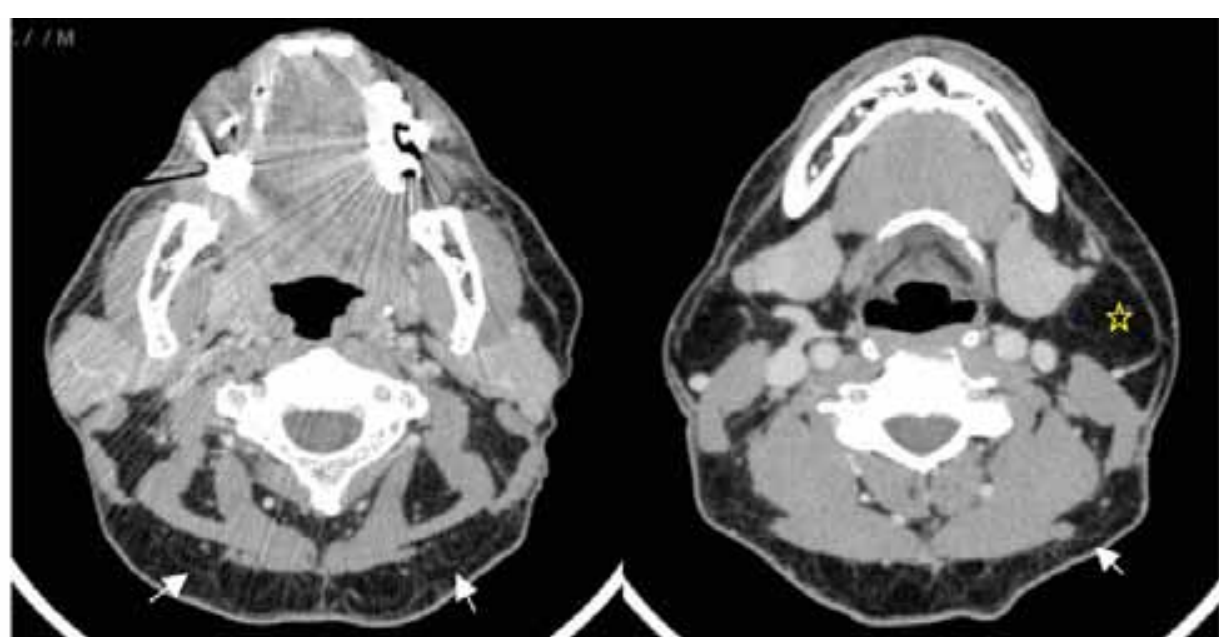

Fig 2A

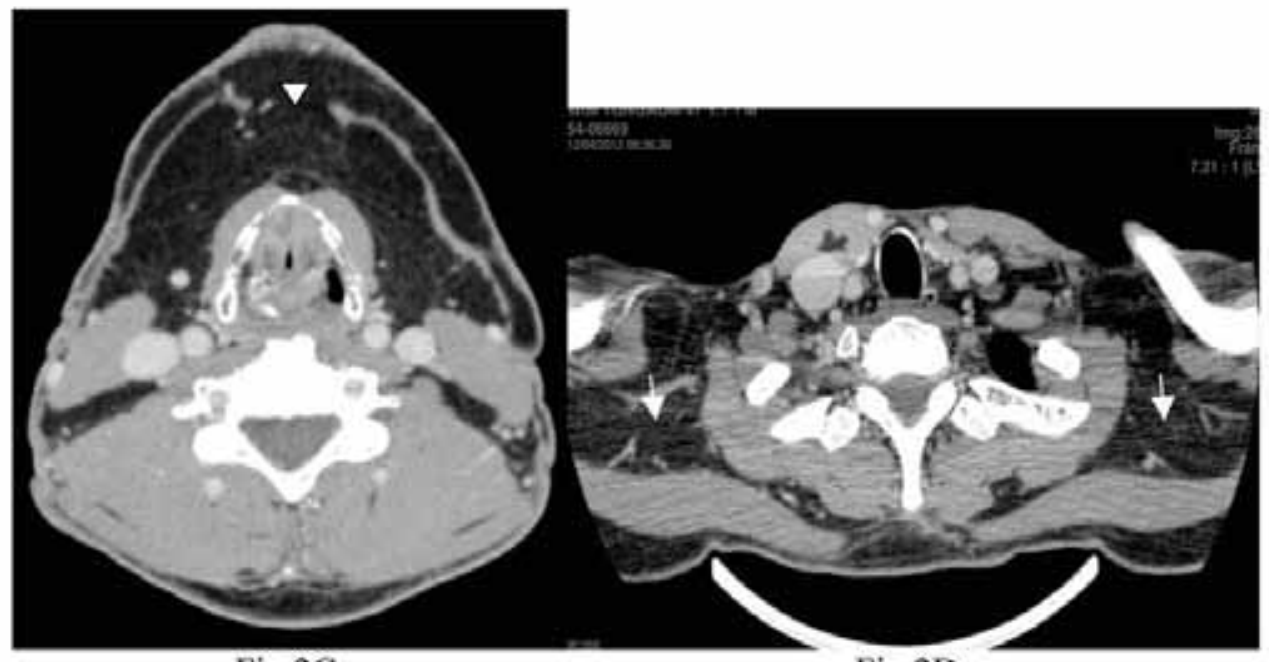

Fig 2C

Fig 2D

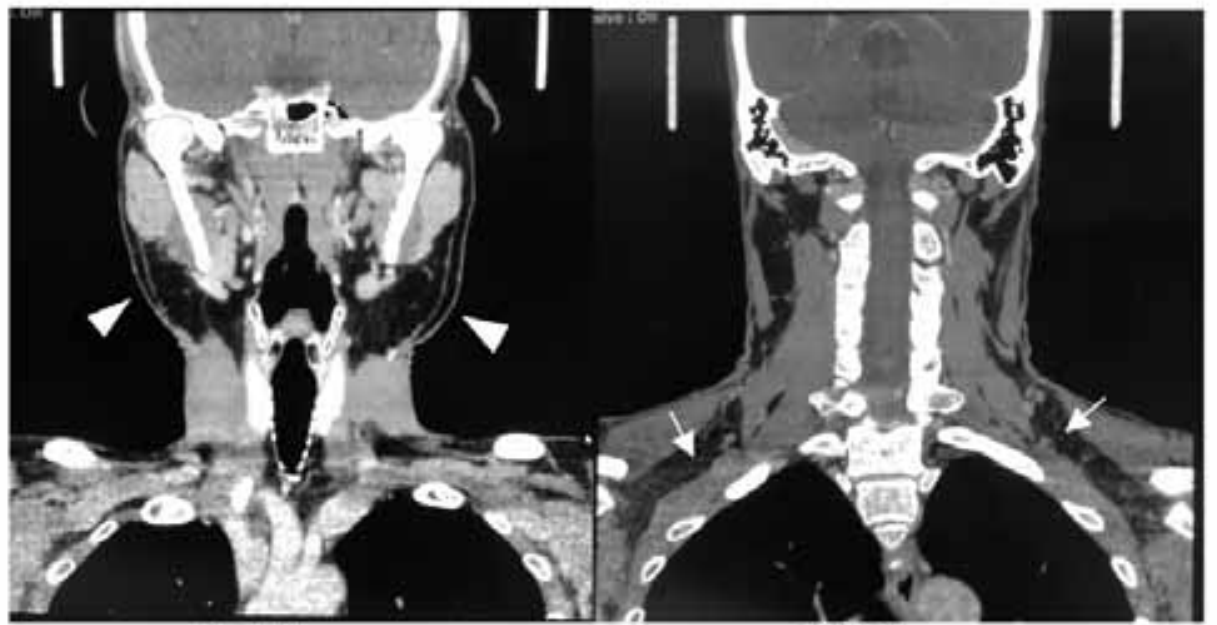

Fig 2E

Fig $2 \mathrm{~F}$

Figure. 2. 2A - 2D Axial CT scan, Figure 2E and 2F Coronal reconstruction MDCT scan of the neck revealed generalized excessive fat density deposition at both cheeks (star), chin (arrow head), paraspinal region and surrounding all anterior, lateral and posterior necks (small white arrow) 


\section{Discussion}

Multiple symmetric lipomatosis was first described in 1888 by the German surgeon Otto Maldelung. Several years later, Lanois and Bensaude described a further case series of the same entity, which is now referred to as Madelung's disease, Launois-Bensaude syndrome, benign symmetric lipomatosis or cephalothoracic lipodystrophy ${ }^{(2,3)}$. This is a rare disorder of unknown cause. In reported case series, up to $90 \%$ of patients have a history of chronic alcoholism, and there is a strong male predominance ${ }^{(4)}$. There is a predilection for people from the Mediterranean ${ }^{(2,5)}$ and not much in oriental countries $^{(6)}$. The signs and symptoms of Madelung's disease vary from person to person. The deposits present in the disease typically occur around the face, the back of the head, the neck, the upper arms, the abdomen, and the back and upper parts of the leg in a very specific pattern or distribution, rarely tongue, mediastinal or below knee involvement occurs. Often, the disease has a period of rapid growth following by a period of stabilization, without spontaneous resolution. Unlike the usual lipoma, these benign fatty masses are not enclosed within a membranous capsule with very distinct boundaries that are often dismissed as simple obesity. It has been divided into three types: Type I or diffuse lipomatosis of the neck (horsecollar lipomata); Type II or multiple symmetric lipomata of the shoulder girdle, the upper arms, the thorax, the thighs and sometimes the abdomen (pseudoathletic appearance); and Type III or a rare type with preponderance of the lipomatosis in the thigh girth (gynecoid type). Women tend to have Type II and $\mathrm{III}^{(7)}$. The most common complaint is the significant cosmetic deformities. However, some patients also complain of movement limitation and neurological dis- turbances such as weakness, areas of paresthesia as well as autonomic nervous system manifestations such as unusual flushing and sweating (particularly after eating), wide fluctuations in blood pressure and heart rate, adult onset asthma, glucose intolerance, gastrointestinal problems (particularly nocturnal diarrhea), difficulty swallowing, hoarseness, sleep problems and foot problems such as ulcers on the plantar surface of the foot or spontaneous fractures of small bones. The only serious condition is laryngeal involvement causing respiratory obstruction. The prognosis is probably more dependent on the associated alcoholism.

The differential diagnosis includes obesity, steroid adiposity, harmartomatous polyposis, adiposis dolorosa (Dercum's disease: multiple painful lipomas) and lipomas. There is also one reported case of spontaneous transformation of Maldelung's disease into liposarcoma. From the radiological point of view, this can be problematic because of difficulties in distinguishing between lipoma and welldifferentiated liposarcoma ${ }^{(2)}$.

The exact cause of Madelung's disease remains unknown, but several theories have been proposed. The condition may also relate to another type of an enzyme defect, or a change in the surface of cells, that prevents the breakdown of fat. Some study suggests that the disease is an endocrine disorder due to inability to properly metabolize fat in affected individuals. One published data suggest that children with similar findings might have an underlying mitochondrial dysfunction ${ }^{(2)}$. There have been some cases reported among women as well as in nonalcoholic males and females. Alcohol consumption may play an important role in any of these potential causes. Blood studies tend to be quite normal. CT scan and MRI demonstrate the pres- 
ence of the fatty masses that helpful in diagnosis. However, biopsy of a fatty mass with underlying muscle tissue, and the identification of specific pathology, is significant in confirming a diagnosis ${ }^{(8)}$.

The most effective treatment for Madelung's disease is surgery (surgical excision and/or liposuction). Liposuction has gained popularity in more recent years due to its minimal scarring, less invasive, technically easier, and better suited for individuals with a higher surgical or anesthetic risk but its limitation for inadequate aspiration of lipoma. Some researchers believe it is unnecessary to take surgical risk because the condition is usually benign. The surgical excision should be limited to those with airway compression or severe cosmetic deformities. Reportedly, it is rarely possible to remove the lipomas completely. Some studies have reported successful treatment with salbutamol, which increases the breakdown of lipids (lipolysis). Abstaining from alcohol intake, weight loss, and correction of any associated metabolic/endocrine abnormalities are recommended ${ }^{(1)}$.

\section{Conclusion}

Madelung's disease is a rare benign slowly progressive disorder but associated with a significant morbidity and mortality, particular respiratory obstruction. Surgical treatment should be performed only in case of airway compression or severe cosmetic deformities. Abstinence from ethanol ingestion and corrected metabolic disorder could relief the affectation.

\section{References}

1. National Center for Advancing Translational Sciences (NCATS). National Institutes of Health (NIH). Genetic and Rare Diseases Information Center. Madelung disease. Available from: http://rarediseases.info.nih.gov/gard/6957/ madelung-disease/resources/1 (http://rarediseases.info. nih.gov/benign symmetrical lipomatosis)

2. Gutzeit A, Binkert C, Schmidt S, Jandali A, Mutschler J, Hergan K, et al. Growing fatty mass in the back: diagnosis of a multiple symmetric lipomatosis (Madelung's disease) in association with chronic alcoholism. Skeletal Radiology April 2012, Volume 41, Issue 4, pp 489-490. Available from: http://link.springer.com/article/10.1007\% 2Fs00256-011-1280-6

3. MedicineNet.com. http://www.medterms.com/script/main/ art.asp?articlekey=9399

4. Ampollini L, M.D., Carbognani P, M.D., Ph.D. Madelung's Disease N Engl J Med 2011; 364:465 February 3, 2011DOI: 10.1056/NEJMicm1003531

5. Bulum T, Duvnjak L, Car N, Metelko Z. MADELUNGีS DISEASE: CASE REPORT AND REVIEW OF THE LITERATURE. Vuk Vrhovac University Clinic, Zagreb, Croatia. Diabetologia Croatica 36-2, 2007

6. ปรีดี เงาเทพพฤฒาราม. พบ. Madelung's Disease (Multiple Symmetric Lipomatosis). J Prapokklao Hosp Clin Med Educat Center. Vol. 25 No. 2 Apr.-Jun. 2008

7. Herbst K, PH.D.,M.D. Madelung's disease http://www. lipomadoc.org/madelungs-disease.html

8. J: Jbenign symmetrical lipomatosis\MSL, Multiple Symmetrical Lipomatosis, also known as Benign Symmetric Lipomatosis or Madelung's Disease and Lanois-Bensaude Syndrome-Cushing's Help and Support.mht, Available from: http://www.cushings-help.com/madelung.htm 